\title{
State-Dependent Gaussian Z-Channel with Mismatched Side-Information and Interference
}

\author{
Ruchen Duan, Yingbin Liang \\ Dept of EECS \\ Syracuse University \\ Syracuse, NY 13244, USA \\ \{rduan,yliang06\}@syr.edu
}

\author{
Ashish Khisti \\ School of ECE \\ University of Toronto \\ Toronto, ON, M5S3G4, Canada \\ akhisti@comm.utoronto.ca
}

\author{
Shlomo Shamai (Shitz) \\ Dept of EE \\ Technion-Israel Institute of Technology \\ Technion city, Haifa 32000, Israel \\ sshlomo@ee.technion.ac.il
}

\begin{abstract}
A state-dependent Gaussian Z-interference channel model is investigated in the regime of high state power, in which transmitters 1 and 2 communicate with receivers 1 and 2 , and only receiver 2 is interfered by transmitter 1's signal and a random state sequence. The state sequence is known noncausally only to transmitter 1 , not to the corresponding transmitter 2 . A layered coding scheme is designed for transmitter 1 to help interference cancelation at receiver 2 (using a cognitive dirty paper coding) and to transmit its own message to receiver 1. Inner and outer bounds are derived, and are further analyzed to characterize the boundary of the capacity region either fully or partially for all Gaussian channel parameters. Our results imply that the capacity region of such a channel with mismatched transmitter-side state cognition and receiver-side state interference is strictly less than that of the corresponding channel without state, which is in contrast to Costa type of dirty channels, for which dirty paper coding achieves the capacity of the corresponding channels without state.
\end{abstract}

\section{INTRODUCTION}

A number of state-dependent interference channel models have recently been intensively studied. These models [1]-[4] are based on the classic interference channel with receivers being interfered not only by other transmitters' signals but also by random state sequences. The state sequences are known noncausally to some or all of the transmitters. Similar models of the state-dependent cognitive interference channel have also been studied in [5]-[8], in which the transmitters and/or receivers have message cognition.

In this paper, we study a state-dependent Z-interference channel (see Fig. 1), in which transmitters 1 and 2 communicate with receivers 1 and 2, and only receiver 2 is interfered by transmitter 1's signal and a random state sequence. In contrast to the state-dependent Z-interference channel model studied previously in [9], which assumes that state interference at both receivers are known to both (corresponding) transmitters, our model assumes that state interference (only to receiver 2 ) is known noncausally only to transmitter 1 , not to the corresponding transmitter 2. Hence, transmitter-side state cognition and receiver-side state interference are mismatched. Our goal is to investigate such a mismatched scenario in high state power regime, i.e., as the power of the state sequence $Q \rightarrow \infty$.

In general, it is challenging to design capacity-achieving schemes for such a system with mismatched property. Clearly, it is not possible for transmitter 1 to directly cancel state interference due to the large state power. One natural idea is to apply lattice coding in high state power regime as in [10] for the state-dependent multiple access channel (MAC). However, lattice coding does not achieve the capacity for our model here. Another approach is to apply dirty paper coding [11]. However, the difficulty associated with the Z-channel here lies in that transmitter 1 needs to resolve the tension between transmitting its own message to receiver 1 and helping receiver 2 to cancel its interference.

In this paper, we design a layered coding scheme, in which a cognitive dirty paper coding scheme for transmitter 1 to help interference cancelation is superposed with transmitter 1's transmission to receiver 1 . It can be shown that when receiver 1 's power is sufficiently large, layered coding we adopt is superior to time-sharing between serving receiver 2 (using cognitive dirty paper coding) and receiver 1 . In our scheme, cognitive dirty paper coding is designed due to mismatched state cognition and interference, in which transmission of the message and treatment of state interference are performed by separate transmitters. Differently from dirty paper coding for the asymmetric MAC in [12], cognitive dirty paper coding does not encode any message, as transmitter 1 does not know message 2 that is interfered. In such a scheme, correlation between the state variable and the state-cancelation variable is a design parameter, and can be chosen to optimize the rate region. This is in contrast to classical dirty paper coding [11], in which such a correlation parameter is fixed for fully canceling the state. We note that such a scheme can be readily applied to study the MAC with two independent states [13] and the relay channel with state cognition [14], [15]. We summarize our main contributions as follows:

- We develop a layered coding approach for the mismatched scenario, based on which we derive achievable regions for both the discrete memoryless and Gaussian dirty (i.e., statedependent) Z-interference channels.

- We derive an outer bound for the Gaussian dirty Zinterference channel in high state power regime. This outer bound (and hence the capacity region) is strictly inside an achievable rate region (and hence the capacity region) of the corresponding Z-interference channel without state [16]. This is in contrast to the results for Costa type of dirty paper channels, for which dirty paper coding achieves the capacity of the corresponding channels without state.

- By comparing the inner and outer bounds, we characterize 
the boundary of the capacity region either fully or partially for all Gaussian channel parameters in high state power regime.

The rest of the paper is organized as follows. In Section II, we describe the channel model. In Section III, we present inner and outer bounds on the capacity region. In Section IV, we characterize the boundary of the capacity region fully or partially for all Gaussian channel parameters. Finally, we conclude the paper in Section V.

\section{ChANNEL MODEL}

We study a state-dependent $Z$-interference channel model (see Fig. 1) defined as follows. Transmitters 1 and 2 wish to send messages $W_{1}$ and $W_{2}$ respectively to receivers 1 and 2. The two messages are assumed to be independent of each other, and are randomly and independently distributed over the message sets $\mathcal{W}_{1}$ and $\mathcal{W}_{2}$, respectively. Encoder 1 at transmitter $1, f_{1}: \mathcal{W}_{1} \times \mathcal{S}^{n} \rightarrow \mathcal{X}_{1}^{n}$, maps each message $w_{1} \in \mathcal{W}_{1}$ and a state sequence $s^{n} \in \mathcal{S}^{n}$ to a codeword $x_{1}^{n} \in \mathcal{X}_{1}^{n}$, where $\mathcal{X}_{1}$ and $\mathcal{S}$ are the channel input and state alphabets, respectively, and $n$ is the number of channel uses. The sequence $s^{n}$ is the realization of an identical and independently distributed (i.i.d.) state sequence $S^{n}$, which is noncausally known only at transmitter 1 , not at any other terminals in the system. Encoder 2 at transmitter 2, $f_{2}: \mathcal{W}_{2} \rightarrow \mathcal{X}_{2}^{n}$, maps each message $w_{2} \in \mathcal{W}_{2}$ to a codeword $x_{2}^{n} \in \mathcal{X}_{2}^{n}$, where $\mathcal{X}_{2}$ is the second channel input alphabet. The two inputs $x_{1}^{n}$ and $x_{2}^{n}$ are transmitted over a Z-interference channel with transition probability having marginal distributions $P_{Y \mid X_{1}}$ and $P_{Z \mid X_{1}, X_{2}, S}$. Two decoders respectively at receivers 1 and 2 , $g_{1}: \mathcal{Y}^{n} \rightarrow \mathcal{W}_{1}$ and $g_{2}: \mathcal{Z}^{n} \rightarrow \mathcal{W}_{2}$, map received sequences $y^{n}$ and $z^{n}$ to estimates $\hat{w}_{1} \in \mathcal{W}_{1}$ and $\hat{w}_{2} \in \mathcal{W}_{2}$, respectively.

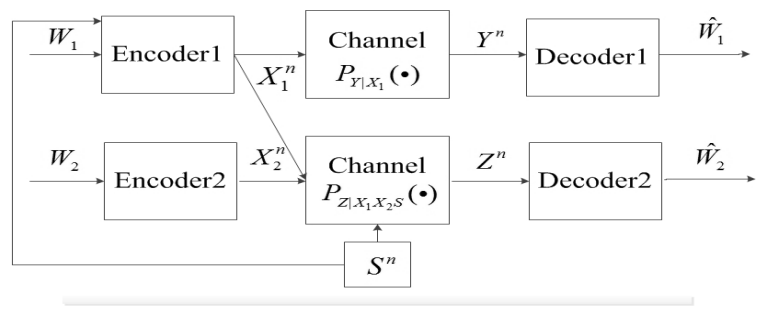

Fig. 1. A state-dependent Z-interference channel model.

The average probability of error for a length- $n$ code is defined as

$$
P_{e}^{(n)}=\frac{1}{\left|\mathcal{W}_{1}\right|\left|\mathcal{W}_{2}\right|} \sum_{w_{1}=1}^{\left|\mathcal{W}_{1}\right|} \sum_{w_{2}=1}^{\left|\mathcal{W}_{2}\right|} \operatorname{Pr}\left\{\left(\hat{w}_{1}, \hat{w}_{2}\right) \neq\left(w_{1}, w_{2}\right)\right\} .
$$

A rate pair $\left(R_{1}, R_{2}\right)$ is achievable if there exists a sequence of message sets $\mathcal{W}_{1 n}$ and $\mathcal{W}_{2 n}$ with $\left|\mathcal{W}_{1 n}\right|=2^{n R_{1}}$ and $\left|\mathcal{W}_{2 n}\right|=$ $2^{n R_{2}}$, and encoder-decoder tuples $\left(f_{1 n}, f_{2 n}, g_{1 n}, g_{2 n}\right)$ such that the average error probability $P_{e}^{(n)} \rightarrow 0$ as $n \rightarrow \infty$. The capacity region is defined to be the closure of the set of all achievable rate pairs $\left(R_{1}, R_{2}\right)$.

In this paper, we focus on the Gaussian channel with the outputs at receivers 1 and 2 for one channel use given by

$$
Y=X_{1}+N_{1}, \quad Z=X_{1}+X_{2}+S+N_{2}
$$

where the noise variables $N_{1}$ and $N_{2}$ and the state variable $S$ are Gaussian distributed with distributions $N_{1}, N_{2} \sim \mathcal{N}(0,1)$ and $S \sim \mathcal{N}(0, Q)$, and all of the three variables are i.i.d. over channel uses. The channel inputs $X_{1}$ and $X_{2}$ are subject to the average power constraints $P_{1}$ and $P_{2}$. Our goal is to characterize the capacity region of the Gaussian channel in high state power regime, i.e., as $Q \rightarrow \infty$.

\section{INNER AND OUTER BOUNDS}

In this section, we provide inner and outer bounds (on the capacity region) for the state-dependent Gaussian Zinterference channel. We characterize the boundary of the capacity region based on these bounds in the next section.

\section{A. Inner Bound}

The major challenge in designing an achievable scheme arises from the mismatched property due to transmitter-side state cognition and receiver-side state interference. Thus, transmitter 1 needs to resolve the tension between transmitting its own message to receiver 1 and helping receiver 2 to cancel its interference. A simple scheme of time-sharing between the two in general is not optimal. In our scheme, transmitter 1 splits its signal into two parts in a layered fashion: one (represented by $X_{1}^{\prime}$ in Lemma 1) for transmitting its own message and the other (represented by $U$ in Lemma 1) for helping receiver 2 to remove both state and signal interference via a single-bin binning scheme. In particular, the second part of the scheme does not apply dirty paper coding [11] (that removes state interference for the encoded message) directly, because transmitter 1 knows the state but does not know the message (of transmitter 2) that the state interferes, and hence cannot encode this message via dirty paper coding. Instead, a cognitive dirty paper coding scheme is applied, in which transmission of $W_{2}$ and treatment of state interference for decoding $W_{2}$ are performed separately by transmitters 2 and 1 . Based on such a scheme, we obtain the following achievable rate region for the discrete memoryless channel, which is useful for deriving an inner bound for the Gaussian channel. The detail of the proof is omitted due to the space limitations.

Lemma 1. For the discrete memoryless state-dependent Zinterference channel defined in Section II, an inner bound on the capacity region consists of rate pairs $\left(R_{1}, R_{2}\right)$ satisfying:

$$
\begin{aligned}
& R_{1} \leqslant I\left(X_{1}^{\prime} ; Y\right) \\
& R_{2} \leqslant I\left(X_{2} ; Z \mid U\right) \\
& R_{2} \leqslant I\left(X_{2} U ; Z\right)-I\left(U ; S X_{1}^{\prime}\right)
\end{aligned}
$$

for some distribution $P_{S} P_{X_{1}^{\prime}} P_{U \mid S X_{1}^{\prime}} P_{X_{1} \mid U S X_{1}^{\prime}} P_{X_{2}} P_{Y Z \mid S X_{1} X_{2}}$.

Based on Lemma 1, we have the following simpler inner bound by adding a constraint to remove (3) as a redundant bound.

Corollary 1. For the discrete memoryless state-dependent Zinterference channel defined in Section II, an inner bound on the capacity region consists of rate pairs $\left(R_{1}, R_{2}\right)$ satisfying:

$$
\begin{aligned}
& R_{1} \leqslant I\left(X_{1}^{\prime} ; Y\right) \\
& R_{2} \leqslant I\left(X_{2} ; Z \mid U\right)
\end{aligned}
$$


for some distribution $P_{S} P_{X_{1}^{\prime}} P_{U \mid S X_{1}^{\prime}} P_{X_{1} \mid U S X_{1}^{\prime}} P_{X_{2}} P_{Y Z \mid S X_{1} X_{2}}$ that satisfies $I(U ; Z) \geqslant I\left(U ; S X_{1}^{\prime}\right)$.

We next derive an inner bound for the Gaussian channel based on Corollary 1 by setting $U=X_{0}+\alpha\left(S+X_{1}^{\prime}\right)$ and $X_{1}=X_{0}+X_{1}^{\prime}$ with independent $X_{0}, X_{1}^{\prime}$ and $X_{2}$ having distributions $X_{0} \sim \mathcal{N}\left(0, \beta P_{1}\right), X_{1}^{\prime} \sim \mathcal{N}\left(0, \bar{\beta} P_{1}\right)$ and $X_{2} \sim \mathcal{N}\left(0, P_{2}\right)$, where $\alpha \geqslant 0,0 \leqslant \beta \leqslant 1$, and $\bar{\beta}=1-\beta$. We note that although Corollary 1 may provide a smaller inner bound than that given in Lemma 1, it can be shown that two inner bounds are equivalent for our chosen auxiliary random variables and input distribution.

Proposition 1. For the state-dependent Gaussian Zinterference channel defined in Section II, an inner bound on the capacity region consists of rate pairs $\left(R_{1}, R_{2}\right)$ satisfying:

$$
\begin{aligned}
& R_{1} \leqslant \frac{1}{2} \log \left(1+\frac{\bar{\beta} P_{1}}{\beta P_{1}+1}\right) \\
& R_{2} \leqslant \frac{1}{2} \log \left(1+\frac{P_{2}}{1+\left(1-\frac{1}{\alpha}\right)^{2} \beta P_{1}}\right)
\end{aligned}
$$

for some real constants $\alpha \geqslant 0$ and $0 \leqslant \beta \leqslant 1$ that satisfy $\alpha^{2}\left(\bar{\beta} P_{1}+Q\right)\left(\beta P_{1}+P_{2}+1\right)-2 \alpha \beta P_{1}\left(\bar{\beta} P_{1}+Q\right)-\beta^{2} P_{1}^{2} \leqslant 0$. As $Q \rightarrow \infty$, the preceding condition becomes $\alpha \leqslant \frac{2 \beta P_{1}}{\beta P_{1}+P_{2}+1}$.

We note that in Proposition 1, the parameter $\alpha$ captures correlation between the state variable $S$ and the auxiliary variable $U$ for dealing with the state, and can be chosen to optimize the rate region. This is in contrast to the classical dirty paper coding [11], in which such correlation parameter is fixed for state cancelation.

\section{B. Outer Bound}

In this subsection, we provide an outer bound on the capacity region in high state power regime, i.e., as $Q \rightarrow \infty$.

Proposition 2. For the state-dependent Gaussian Zinterference channel defined in Section II, an outer bound on the capacity region for the regime when $Q \rightarrow \infty$ consists of rate pairs $\left(R_{1}, R_{2}\right)$ satisfying:

$$
\begin{aligned}
R_{2} & \leqslant \frac{1}{2} \log \left(1+P_{2}\right) \\
R_{1}+R_{2} & \leqslant \frac{1}{2} \log \left(1+P_{1}\right) .
\end{aligned}
$$

The bound (7) on $R_{2}$ follows simply from the capacity of the point-to-point channel between transmitter 2 and receiver 2 without signal and state interference. The bound (8) on the sum rate is limited only by the power $P_{1}$ of transmitter 1 , and does not depend on the power $P_{2}$ of transmitter 2. Intuitively, this is because $P_{1}$ is split for transmission of $W_{1}$ and for helping transmission of $W_{2}$ by removing state interference, and hence $P_{1}$ determines a trade-off between $R_{1}$ and $R_{2}$. On the other hand, improving the power $P_{2}$, although may improve $R_{2}$, can also cause more interference for receiver 2 to decode the auxiliary variable for canceling interference. Thus, the balance of the two effects turns out not to affect the sum rate. The mathematical proof of the bound (8) is omitted due to the space limitations.
We further note that although the sum-rate upper bound (8) can be achieved easily by keeping transmitter 2 silent (i.e., $R_{1}$ achieves the sum rate bound with $R_{2}=0$ ), we are interested in characterizing the capacity region (i.e., the trade-off between $R_{1}$ and $R_{2}$ ) rather than a single point that achieves the sumrate capacity. In the next section, we characterize such optimal trade-off based on the sum-rate bound.

Remark 1. The outer bound in Proposition 2 is strictly inside an achievable rate region of the corresponding Z-interference channel without state interference [16], which implies that the capacity region of our model is strictly inside that of the corresponding channel without state. This suggests that state interference does cause performance degradation for systems with mismatched state cognition and interference in high state power regime. This is in contrast to the results for Costa type of dirty paper channels, for which dirty paper coding achieves the capacity of the corresponding channels without state.

\section{BOUNDARY OF CAPACITY REgION}

In this section, we characterize the boundary points of the capacity region for the Gaussian channel based on the inner and outer bounds given in Propositions 1 and 2, respectively. We partition the Gaussian channel into four cases based on the conditions on the power constraints: (1) $P_{2} \geqslant P_{1}+1$; (2) $P_{1} \leqslant P_{2}<P_{1}+1$; (3) $P_{1}-1 \leqslant P_{2}<P_{1}$; and (4) $0 \leqslant P_{2}<P_{1}-1$. For each case, we optimize the dirty paper coding parameter $\alpha$ that satisfies $0 \leqslant \alpha \leqslant \frac{2 \beta P_{1}}{\beta P_{1}+P_{2}+1}$ to find achievable rate points that lie on the sum-rate upper bound (8) in order to characterize the boundary points of the capacity region. The proofs are omitted due to the space limitations.

Case 1: $P_{2} \geqslant P_{1}+1$. For this case, we set the actual transmission power for transmission of $W_{2}$ to be $\tilde{P}_{2}=\beta P_{1}+$ 1 , and then the inner bound (6) on $R_{2}$ is optimized when $\alpha=\frac{2 \beta P_{1}}{\beta P_{1}+\tilde{P}_{2}+1}$. Thus, the inner bound given in Proposition 1 matches the outer bound given in Proposition 2, and the capacity region is fully characterized as illustrated in Fig. 2.

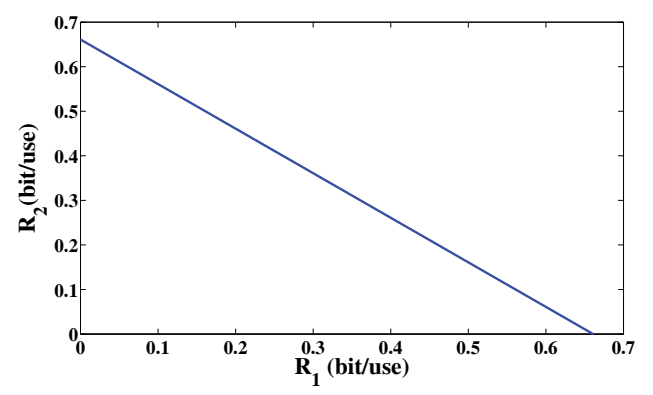

Fig. 2. The capacity region for case 1 with $P_{1}=1.5$ and $P_{2}=3$.

Theorem 1. Consider the state-dependent Gaussian Zinterference channel defined in Section II in the regime when $Q \rightarrow \infty$. If $P_{2} \geqslant P_{1}+1$, the capacity region consists of the rate pairs $\left(R_{1}, R_{2}\right)$ satisfying

$$
R_{1}+R_{2} \leqslant \frac{1}{2} \log \left(1+P_{1}\right) .
$$


Theorem 1 implies that when $P_{2}$ is large enough, the power of transmitter 1 limits the system performance. Furthermore, since $P_{2}$ for transmission of $W_{2}$ causes interference for receiver 2 to decode the auxiliary variable for interference cancelation, beyond a certain value, increasing $P_{2}$ does not improve the rate region any more. Theorem 1 also suggests that in order to achieve different points on the boundary of the capacity region (captured by parameters $\beta$ ), different amounts of power $\widetilde{P}_{2}$ should be applied.

Case 2: $P_{1} \leqslant P_{2}<P_{1}+1$. For this case, if $P_{2}<1$, the inner and outer bounds match only at the rate point $A$ as illustrated in Fig. 3 (a), which achieves the sum-rate capacity. However, if $P_{2} \geqslant 1$, i.e., $P_{2}$ is larger than the noise power, inner and outer bounds match over the line between the points A and B as illustrated in Fig. 3 (b), and thus optimal trade-off between $R_{1}$ and $R_{2}$ is achieved over the points on this line. We summarize this capacity result in the following theorem.

Theorem 2. Consider the state-dependent Gaussian Zinterference channel defined in Section II in the regime when $Q \rightarrow \infty$. If $P_{1} \leqslant P_{2}<P_{1}+1$ and $P_{2} \geqslant 1$, the rate points $\left(R_{1}, R_{2}\right)$ on the line between $\left(\frac{1}{2} \log \left(1+P_{1}\right), 0\right)$ (i.e., point $A$ in Fig. $3(b))$ and $\left(\frac{1}{2} \log \left(1+\frac{P_{1}-P_{2}+1}{P_{2}}\right), \frac{1}{2} \log P_{2}\right)$ (i.e., point $B$ in Fig. $3(b))$ are on the boundary of the capacity region.

Case 3: $P_{1}-1 \leqslant P_{2}<P_{1}$. For this case, similar to case 2, if $P_{2}<1$, the inner and outer bounds match only at point $\mathrm{A}$ as illustrated in Fig. 4 (a), which achieves the sum-rate capacity. However, if $P_{2} \geqslant 1$, inner and outer bounds match over the line between points A and B as illustrated in Fig. 4 (b), and this capacity result is stated in the following theorem.

Theorem 3. Consider the state-dependent Gaussian Zinterference channel defined in Section II in the regime when $Q \rightarrow \infty$. If $P_{1}-1 \leqslant P_{2}<P_{1}$ and $P_{2} \geqslant 1$, the points $\left(R_{1}, R_{2}\right)$ on the line between $\left(\frac{1}{2} \log \left(1+P_{1}\right), 0\right)$ (i.e., point $A$ in Fig. $4(b))$ and $\left(\frac{1}{2} \log \left(1+\frac{P_{1}-P_{2}+1}{P_{2}}\right), \frac{1}{2} \log P_{2}\right)$ (i.e., point B in Fig. $4(b))$ are on the boundary of the capacity region.

We note that case 3 differs from case 2 in that the outer bound for case 3 includes one more bound $R_{2} \leq \frac{1}{2} \log \left(1+P_{2}\right)$. As illustrated in Fig. 4, this upper bound is not achieved by the inner bound, because the power $P_{1}$ is not large enough to perfectly cancel state and signal interference at receiver 2 .

Case 4: $P_{2}<P_{1}-1$. Similar to cases 2 and 3, the inner and outer bounds match partially over the sum rate bound, i.e., the two bounds match at the point A (see Fig. 5 (a)) if $P_{2}<1$, and match over the line between points $A$ and $B$ (see Fig. 5 (b)) if $P_{2} \geqslant 1$. However, differently from case 3 , the inner and outer bounds also match when $R_{2}=\frac{1}{2} \log \left(1+P_{2}\right)$ over the line between points D and E (see Fig. 5 (a) and (b)). This is because the power $P_{1}$ of transmitter 1 in this case is large enough to fully cancel state and signal interference so that transmitter 2 is able to reach its maximum point-to-point rate to receiver 2 without interference. Furthermore, transmitter 1 is also able to simultaneously transmit its own message at a certain positive rate as reflected by the line D-E in Fig. 5 (a) and (b). We summarize these results on the boundaries of the capacity region in the following theorem.

Theorem 4. Consider the state-dependent Gaussian Zinterference channel defined in Section II in the regime when $Q \rightarrow \infty$. If $P_{2}<P_{1}-1$ and $P_{2}<1$, then the point $\left(\frac{1}{2} \log \left(1+P_{1}\right), 0\right)$ (i.e., point $A$ in Fig. $\left.5(a)\right)$ and the points on the line between $\left(\frac{1}{2} \log \left(\frac{P_{1}+1}{P_{2}+2}\right), \frac{1}{2} \log \left(1+P_{2}\right)\right)$ (i.e., point $D$ in Fig. $5(a))$ and $\left(0, \frac{1}{2} \log \left(1+P_{2}\right)\right.$ ) (i.e., point $E$ in Fig. 5 (a)) are on the boundary of the capacity region.

If $P_{2}<P_{1}-1$ and $P_{2} \geqslant 1$, then the points on the line between $\left(\frac{1}{2} \log \left(1+P_{1}\right), 0\right)$ (i.e., point $A$ in Fig. $5(b))$ and $\left(\frac{1}{2} \log \left(1+\frac{P_{1}-P_{2}+1}{P_{2}}\right), \frac{1}{2} \log P_{2}\right)$ (i.e., point $B$ in Fig. $5(b))$, and the points on the line between $\left(\frac{1}{2} \log \left(\frac{P_{1}+1}{P_{2}+2}\right), \frac{1}{2} \log \left(1+P_{2}\right)\right)$ (i.e., point $D$ in Fig. $\left.5(b)\right)$ and $\left(0, \frac{1}{2} \log \left(1+P_{2}\right)\right)$ (i.e., point $E$ in Fig. $\left.5(b)\right)$ are on the boundary of the capacity region.

\section{CONCLUSION}

In this paper, we have studied the state-dependent Gaussian Z-interference channel with mismatched transmitter-side state cognition and receiver-side state interference in high state power regime. We have applied a layer coding scheme with a cognitive dirty paper coding in order to deal with the mismatch property of the channel. We have derived inner and outer bounds on the capacity region for the Gaussian channel, and have shown that the two bounds match either fully or partially for all Gaussian channel parameters. We have also discussed the properties of these rate regions and their implications on the capacity results. In the future, we will further explore other multiuser state-dependent channels with state cognition.

\section{ACKNOWLEDGMENT}

The work of R. Duan and Y. Liang was supported by a NSF CAREER Award under Grant CCF-10-26565 and by the NSF under Grants CCF-10-26566 and CNS-11-16932. The work of A. Khisti was supported by the Canada Research Chair's Program. The work of S. Shamai (Shitz) was supported by the Israel Science Foundation (ISF), and the European Commission in the framework of the Network of Excellence in Wireless COMmunications NEWCOM\#.

\section{REFERENCES}

[1] L. Zhang, J. Jiang, and S. Cui, "Gaussian interference channel with state information," To appear in IEEE Trans. Wireless Commun., 2013.

[2] L. Zhang, T. Liu, and S. Cui, "Symmetric Gaussian interference channel with state information," in Proc. 49th Annual Allerton Conference on Communication, Control, and Computing (Allerton), Sep. 2011.

[3] S. Ghasemi-Goojani and H. Behroozi, "On the achievable rateregions for state-dependent Gaussian interference channel," Available at http://arxiv.org/abs/1301.5535, submitted in January 2013.

[4] R. Duan, Y. Liang, and S. Shamai (Shitz), "On the capacity region of gaussian interference channels with state. To appear in Proc. IEEE Int. Symp. Information Theory (ISIT)," Istanbul, Turkey, Jul. 2013.

[5] A. Somekh-Baruch, S. Shamai (Shitz), and S. Verdú, "Cognitive interference channels with state information," in Proc. IEEE Int. Symp. Information Theory (ISIT), Toronto, Canada, July 2008. 


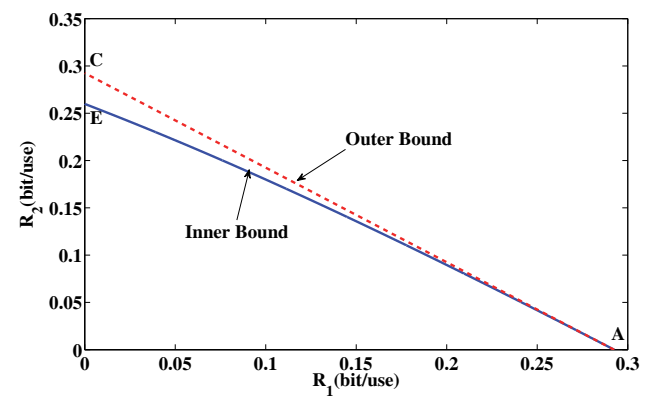

(a) $P_{2}<1$ with $P_{1}=0.5$ and $P_{2}=0.8$

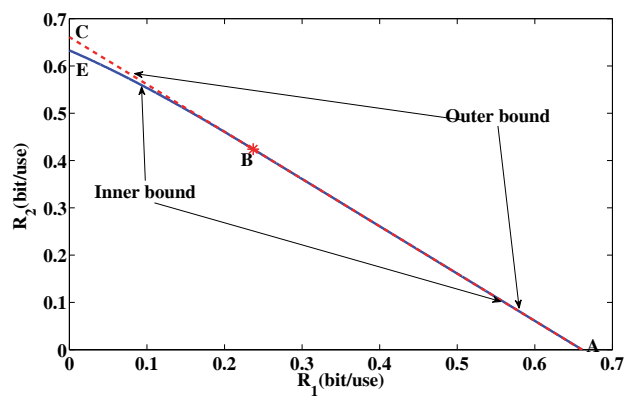

(b) $P_{2} \geqslant 1$ with $P_{1}=1.5$ and $P_{2}=1.8$

Fig. 3. Inner and outer bounds for case 2, which match partially on the boundaries

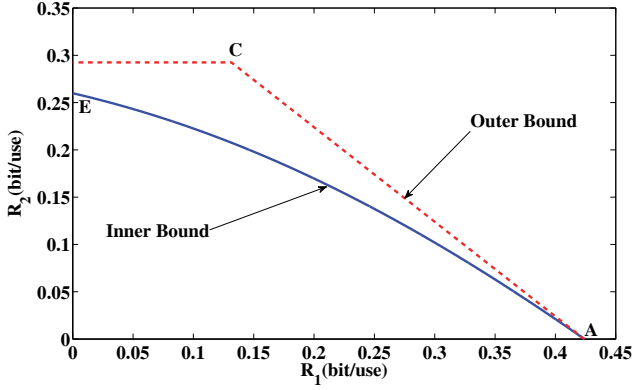

(a) $P_{2}<1$ with $P_{1}=0.8$ and $P_{2}=0.5$

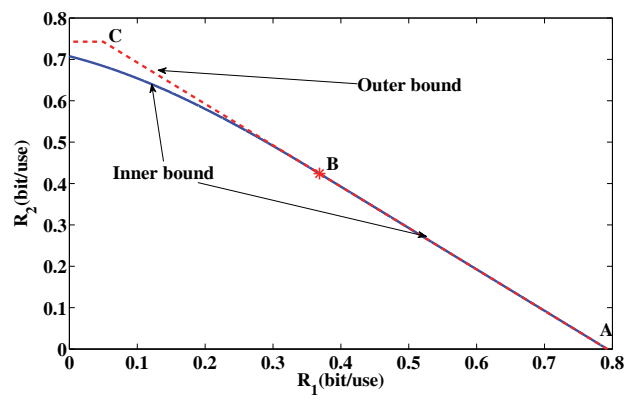

(b) $P_{2} \geqslant 1$ with $P_{1}=2$ and $P_{2}=1.8$

Fig. 4. Inner and outer bounds for case 3 , which match partially on the boundaries

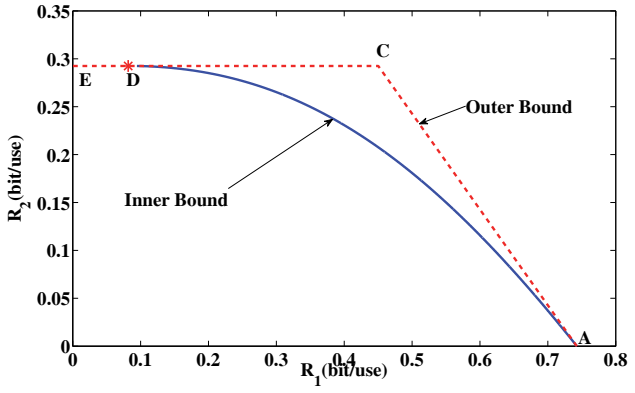

(a) $P_{2}<1$ with $P_{1}=1.5$ and $P_{2}=0.5$

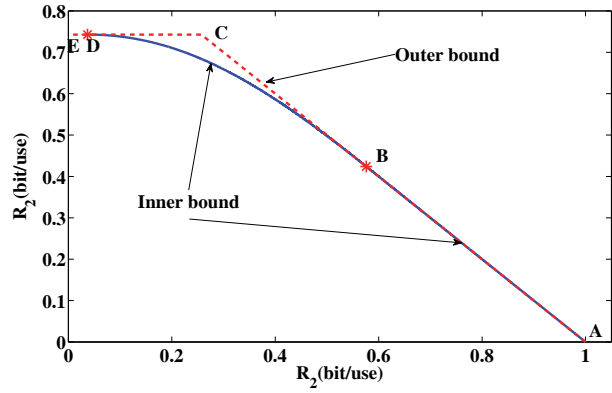

(b) $P_{2} \geqslant 1$ with $P_{1}=3$ and $P_{2}=1.8$

Fig. 5. Inner and outer bounds for case 4, which match partially on the boundaries

[6] R. Duan and Y. Liang, "Gaussian cognitive interference channels with state," in Proc. IEEE Int. Symp. Information Theory (ISIT), Boston, MA, Jul. 2012.

[7] _ "Bounds and capacity theorems for cognitive interference channels with state," Submitted to IEEE Trans. Inform. Theory, June 2012.

[8] M. Kazemi and A. Vosoughi, "On the capacity of the state-dependent cognitive interference channel. To appear in Proc. IEEE Int. Symp. Information Theory (ISIT)," Istanbul, Turkey, Jul. 2013.

[9] S. Hajizadeh, M. Monemizadeh, E. Bahmani, G. A. Hodtani, and M. Joneidi, "State-dependent z channel," Available at http://arxiv.org/abs/1301.6272.

[10] T. Philosof, R. Zamir, U. Erez, and A. J. Khisti, "Lattice strategies for the dirty multiple access channel," IEEE Trans. Inform. Theory, vol. 57, no. 8, pp. 5006-5035, August 2011.

[11] M. H. M. Costa, "Writing on dirty paper," IEEE Trans. Inform. Theory, vol. 29, no. 3, pp. 439-441, May 1983.
[12] S. P. Kotagiri and J. N. Laneman, "Multiaccess channels with state known to some encoders and independent messages," EURASIP Journal on Wireless Communications and Networking, 2008.

[13] I.-H. Wang, "Distributed interference cancellation in multiple access channels," IEEE Trans. Inform. Theory, vol. 58, no. 5, pp. 2781-2787, May 2012.

[14] B. Akhbari, M. Mirmohseni, and M. R. Aref, "Compress-and-forward strategy for the relay channel with non-causal state information," in Proc. IEEE Int. Symp. Information Theory (ISIT), Seoul, Korea, Jul. 2009.

[15] A. Zaidi, S. Shamai (Shitz), P. Piantanida, and L. Vandendorpe, "Bounds on the capacity of the relay channel with noncausal state at source," Submitted to IEEE Trans. Inform. Theory, April 2011.

[16] I. Sason, "On achievable rate regions for the Gaussian interference channel," IEEE Trans. Inform. Theory, vol. 50, no. 6, pp. 1345-1356, June 2004. 